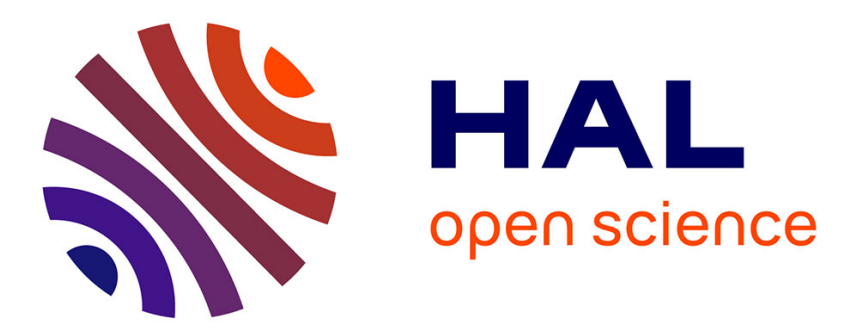

\title{
Remote microscale teleoperation through virtual reality and haptic feedback
}

\author{
Aude Bolopion, Christian Stolle, Robert Tunnell, Sinan Haliyo, Stéphane \\ Régnier, Sergej Fatikow
}

\section{- To cite this version:}

Aude Bolopion, Christian Stolle, Robert Tunnell, Sinan Haliyo, Stéphane Régnier, et al.. Remote microscale teleoperation through virtual reality and haptic feedback. 2011 IEEE/RSJ International Conference on Intelligent Robots and Systems (IROS 2011), Sep 2011, San Francisco, United States. pp.894-900, 10.1109/IROS.2011.6094453 . hal-02912235

\section{HAL Id: hal-02912235 \\ https://hal.science/hal-02912235}

Submitted on 5 Aug 2020

HAL is a multi-disciplinary open access archive for the deposit and dissemination of scientific research documents, whether they are published or not. The documents may come from teaching and research institutions in France or abroad, or from public or private research centers.
L'archive ouverte pluridisciplinaire HAL, est destinée au dépôt et à la diffusion de documents scientifiques de niveau recherche, publiés ou non, émanant des établissements d'enseignement et de recherche français ou étrangers, des laboratoires publics ou privés. 


\title{
Remote Microscale Teleoperation through Virtual Reality and Haptic Feedback
}

\author{
Aude Bolopion ${ }^{*, 1}$, Christian Stolle ${ }^{*, 2}$, Robert Tunnell ${ }^{*, 2}$, Sinan Haliyo ${ }^{1}$, Stéphane Régnier ${ }^{1}$ and Sergej Fatikow ${ }^{2}$
}

\begin{abstract}
This paper reports the remote handling of microscale objects, between two sites approximately $630 \mathrm{~km}$ distant. To manipulate objects less than $10 \mu \mathrm{m}$, specific equipments such as AFM (Atomic Force Microscope) cantilevers integrated into a SEM (Scanning Electron Microscope) are generally required. Enabling remote access to such a system would benefit any micro/nanoresearcher. However, vision feedback and sensor data of a micromanipulation system are generally limited, hence the implementation of a teleoperation scenario is not straightforward. Specific tools are proposed here for an intuitive manipulation in a wide range of applications. To ensure ease of manipulation, both a 3D virtual representation of the scene and haptic feedback are provided. Force sensor feedback is limited since only two measures are available. In order to extend this information, vision algorithms are developed to estimate the respective positions of the tool and objects, which are then used to calculate the haptic feedback. The stability of the overall scheme is very sensitive to time delays. This requirement is taken into account in vision algorithms and the communication module which transfers the data between the two remote sites. In addition, the proposed robotic control architecture is modular so that the platform can be used for a wide range of applications. First results are obtained on a teleoperation between Paris, France, and Oldenburg, Germany.
\end{abstract}

\section{INTRODUCTION}

Teleoperation offers the possibility to perform remote manipulations. In particular it is widely used to interact with hostile environments [1], [2]. Another advantage is to allow a highly trained operator to remotely perform the operation. This case is of particular importance in surgical procedures [3]. Researchers in the field of micro and nanomanipulation would also benefit from these systems which would allow them to access rare and specific equipments such as AFM (Atomic Force Microscope) cantilevers integrated into a SEM (Scanning Electron Microscope).

Intuitivity of such a system is a key parameter for their use in a wide variety of applications and by a large number of researchers. Visual feedback is thus a first requirement. Providing haptic feedback is also a promising solution as it enables operators to feel interactions between the tool

\footnotetext{
${ }^{*}$ A. Bolopion, C. Stolle and R. Tunnell contributed equally to this work.

${ }^{1}$ Authors are with Institut des Systèmes Intelligents et de Robotique, Université Pierre et Marie Curie - Paris 6, CNRS UMR 7222, 4 Place Jussieu, 75005 Paris, France. \{bolopion, haliyo, regnier\}@isir.upmc. fr

${ }^{2}$ Authors are with Division Microrobotics and Control Engineering, Oldenburg University, Oldenburg, Germany. christian.stolle@uni-oldenburg.de
}

and the rest of the manipulation zone, including the to be manipulated objects [4], [5].

For the visual feedback, images from microscopes are commonly used. To provide effective visual feedback virtual reconstruction of the scene can be provided in addition to optical or scanning electron microscope images [6]. However, most of these displays are only 2D [7]. 3D reconstruction would provide a better immersion of the user on the scene.

To compute haptic feedback, information about the scene must be known. However, force sensing is limited since when using a cantilever tool, only two deformations measurements are available [8]. To provide 3D haptic feedback several solutions have been proposed. In particular, physics models are used [9], [10], [11]. Alternatively, strategies are defined from the available measurements to compute haptic guides to perform a given task [12], [13]. However, most of these methods either assume precise knowledge about the environment, or are specific to a particular task. The solution proposed here is to use vision tracking [14] to update a virtual reconstruction of the scene in real-time. The image patterns required for tracking are provided during the system calibration phase in a teach in process.

Another concern is the stability of the teleoperation system. Haptic coupling schemes' stability is highly sensitive to time delays [15]. Solutions based on passivity have been proposed, but are not optimal for microscale since they deteriorate transparency [11]. The whole system should thus be designed to minimize time delays.

Several issues must thus be addressed to provide new tools for intuitive manipulation for a wide range of applications:

1) the robotic control architecture must be organized to enable a wide variety of applications, and should minimize the communication delays,

2) the vision algorithm must provide information about the scene, and be adapted to SEM images. The update rate must be compatible with haptic stability requirements,

3) the vision data should not saturate the network by heavy load transmission, and users should be provided with an intuitive 3D display of the scene,

4) the haptic feedback must be designed according to available information, and the coupling should be transparent and stable.

These issues are addressed on this paper. This work is based on previous studies on the design of the experimental setup 
[16] and the software architecture [17] and on analyzes of haptic coupling schemes [18].

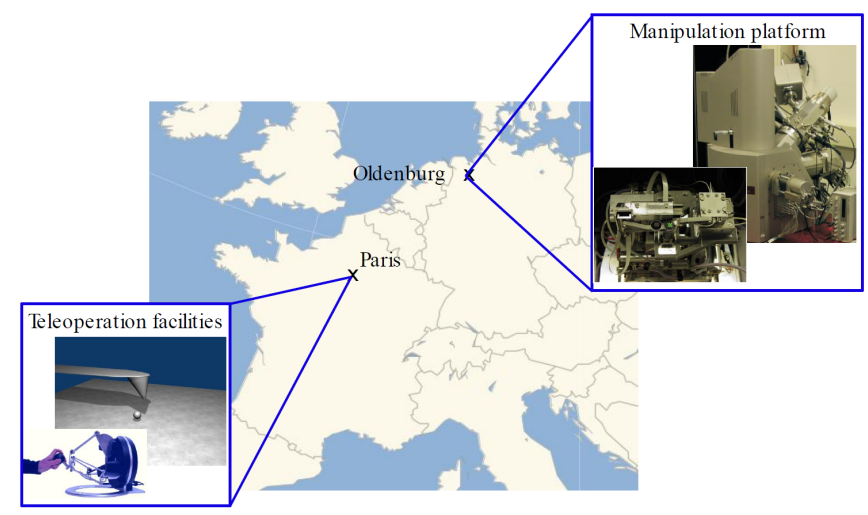

Fig. 1. Teleoperation between Paris, France, and Oldenburg, Germany (630 km point-to-point distance).

The remote handling is implemented between the Microrobotics and Control Engineering division (AMIR) of Univ. Oldenburg, Germany and the Institut des Systèmes Intelligents et de Robotique (ISIR) of Univ. Pierre et Marie Curie, Paris, France. The micromanipulation setup, situated in Oldenburg, is composed of an AFM (Atomic Force Microscopy) manipulator integrated in an SEM to combine the performances of cantilever based manipulation to the vision capabilities of scanning electron microscopes [16]. So that researchers get access to this rare equipment, a remote teleoperation system is implemented in Paris, where microscale teleoperation is a well studied topic [13]. This platform is composed of a haptic interface and a virtual reality room.

This paper is organized as follows. The experimental setup is presented in Section II. Section III details the robotic control architecture. Algorithms for vision detection and tracking are detailed in Section IV. These data are used to determine the haptic feedback and the update of the virtual scene as detailed in Section V. Experimental results are given in Section VI. Section VII concludes the paper.

\section{Microscale Teloperation System}

The micromanipulation setup is composed of a commercially available piezoresistive contact type cantilever ${ }^{1}$ with a stiffness of 4 N.m ${ }^{-1}$. Vertical forces applied on the cantilever are translated into a change in resistance and measured as voltage change. Voltage measurements are made using an amplifier $^{2}$ and a 16-bit analog digital converter ${ }^{3}$. Due to the design of the cantilever, the resolution of torsional forces is low, and do not provide accurate enough information to compute haptic feedback. SEM images will be used to compensate for this lack of force measurement. To increase

\footnotetext{
${ }^{1}$ SSI-SS-ML-PRC400, Seiko Instruments

${ }^{2}$ Hottinger Baldwin Messtechnik GmbH

${ }^{3}$ NI-PCI-6259, National Instruments
}

the field of view, the head part of the cantilever has been cut thinner by a focused ion beam (FIB).

The cantilever is used to manipulate commercially available melanin microspheres ${ }^{4}$ (diameter: $1-2 \mu \mathrm{m}$ ) loosely deployed on a silicon plate $(5 \mathrm{~mm} \times 5 \mathrm{~mm})$. It is mounted on the fine positioning part of a nanorobotic setup [16]. This positioning part is piezo driven with a build-in capacitive position sensors for closed-loop movement control and high accuracy positioning 5 . Its lateral stroke is up to $100 \mu \mathrm{m}$ and the z-range is up to $50 \mu \mathrm{m}$ and the positioning accuracy is at the lower nanometer scale. The specimen holder has been mounted on a coarse positioning unit. This unit consists of three slip stick driven, orthogonal mounted linear axes ${ }^{6}$. These positioners are equipped with optical positioning sensors allowing for travel ranges of several centimeters with a repositioning accuracy up to $50 \mathrm{~nm}$.

The whole setup is placed in a high-resolution scanning electron microscope with a Schottky-emitter ${ }^{7}$ that is also equipped with a FIB column ${ }^{8}$ and a gas injection system.

Researchers are situated in Paris (France). They teleoperate the cantilever through an Omega haptic device ${ }^{9}$. This master arm is a 3 degrees of freedom device for both displacement and haptic feedback. To avoid damages of the haptic interface forces higher than $3 \mathrm{~N}$ are truncated. Users are immersed in a virtual reality room which comprises a 3D display module in addition to the haptic device. A reconstructed image of the scene is projected in stereoscopic $3 \mathrm{D}$ on a $1.80 \times 2.4 \mathrm{~m}^{2}$ screen.

Position and force data are exchanged between the two distant sites by a communication module described in Section III.

\section{REMOTE COMMUNiCATION AND SOFTWARE ARCHITECTURE}

In order that the teleoperation system remains stable, the robotic control system must be designed to minimize time delays. It should also be modular to enable a wide variety of applications. To meet these requirements, it is based on the distributed control system for automated micro- and nano robotics (DCAAN) [17]. It consists in several servers written in $\mathrm{C}++$ which are connected via CORBA (Common Object Request Broker Architecture) based on a TCP/IP network. The servers are categorized into three roles, low level control server (Lolec), sensor provider (Sepro) and a high level control program (Hilec). Lolecs are responsible for executing automation process primitives for a single robot such as "move to position xy". Sepros provide sensor data to all Lolecs or Hilecs on a push basis. A Sepro sends new data as soon as it is available or at a minimum update

\footnotetext{
${ }^{4}$ Micro Particles GmbH

${ }^{5}$ Physikinstrumente $\mathrm{GmbH}$

${ }^{6}$ SmarAct $\mathrm{GmbH}$

${ }^{7}$ Lyra2, Tescan

${ }^{8}$ Canion, Orsay-Physics

${ }^{9}$ Force Dimension, http://www.forcedimension.com
} 
interval. Hilec [19] is an automation server, which processes Python-based scripts. In these automation scripts the process primitives offered by all connected Lolecs and Sepros can be used.

Due to the distributed nature of the tele-control task the software has been subdivided into two parts (see Fig. 2). The one located in Paris is the operator side, and the one in Oldenburg is the manipulation setup. At the remote side three different programs are running:

- the Sepro Olvis is responsible for processing image based information such as video streams [20]. The Olvis framework runs on a Quad Core I7 Intel computer. It is directly connected via local area network with the SEM control computer in Oldenburg. To ensure modularity, it can be configured by combining algorithms in a graph like structure. Depending on these algorithms different kinds of information such as sphere and cantilever positions can be derived (see Section IV). Olvis and Hilec share a common graphical user interface (GUI) for displaying image processing graphs and live images at various processing stages. The GUI also provides all functions of an integrated development environment for automation scripts,

- the AFMLolec is processing the tele-control commands by moving the cantilever tip to the target position and sending an estimation of the actual position and a measure of the force back in return. The control of the AFM itself is realized in hardware [16]. The AFMLolec is capable of processing primitives and translates them into hardware control signals,

- the SEMLolec enables in particular the operator to turn the experimental setup towards the focus of the SEM by moving the SEM stage, and to change image parameters such as brightness and contrast. These settings are indeed of primary importance to ensure efficient vision tracking.

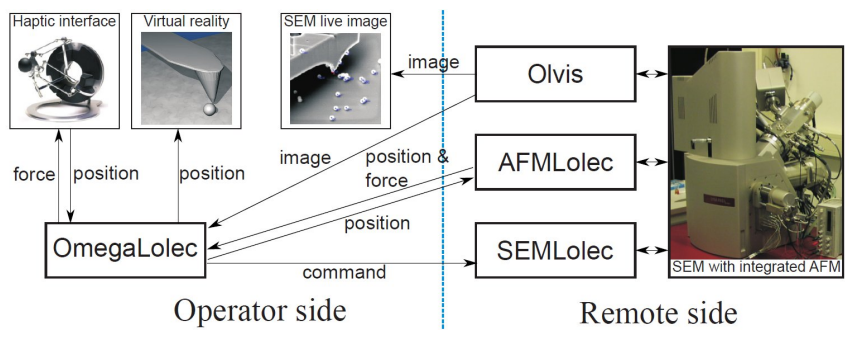

Fig. 2. Software architecture and communication layout between the remote side in Oldenburg and the operator side in Paris

At the operator side the virtual reality server and the OmegaLolec is located. The OmegaLolec server forms the heart of the operator side software and provides a small user interface which enables the operator to initialize and calibrate the system as well as to monitor information such as force and position of the cantilever. In addition to the numerical display a live SEM image (see Section
IV) can be provided to the operator via the GUI. The OmegaLolec is also responsible for translating the force and position feedback of the AFM-cantilever and forces and positions of the haptic device into each other. The positional updates of the cantilever and the sphere are managed by the OmegaLolec.

The communication between the operator and the remote sides has been realized directly via UDP packages. The communication delay has been measured by the means of the round trip time (RTT) by requesting the current position of the cantilever 100 times. The average RTT has been determined at $37 \mathrm{~ms}$, the minimum RTT at $36 \mathrm{~ms}$ and the maximum RTT at $39 \mathrm{~ms}$. The distributed control system enables to minimize RTT, and to ensure modularity. It is thus suitable to perform a wide range of teleoperation over important distances.

\section{IMAge PROCESSING AND TRACKING}

Due to the lack of force measurement, images from the SEM are used to get information about the scene. Tracking algorithms must be adapted to the specificity of SEM images, and the update rate must be sufficient to meet the requirements of haptic feedback. Images are acquired with a resolution of $627 \times 627$ pixels. The view field of the handling scene is in the range of 10 to $100 \mu \mathrm{m}$.

The cantilever is tracked with a template matching algorithm (TM) [21], which is one of the fastest and the most common real-time marker tracking approaches. It finds the position in the image with the best correspondence with the template. To achieve good tracking requirements, a mark has been structured by the FIB at the top of the cantilever (Fig. 3(a)). This mark is used as the template for the TM, and its unique features enable to precisely determine the position of the cantilever. In the initialization step the mark is tagged manually. Automated detection will be considered in future works. To calculate the position of the cantilever tip, a offset is added to the position of the tracking mark. To be used in teleoperation, the update rate of the position of the cantilever has to be as fast as possible. Therefore the process is set to the highest priority in the Olvis framework to achieve an update rate of up to $50 \mathrm{~Hz}$.

The melanin spheres are tracked with a cross correlation (CC) based template matching approach. This algorithm is developed to especially meet the requirements of object tracking in the SEM. In particular, the algorithm integrates SEM parameters like view field to achieve a magnification invariant tracking. The tracking of the melanin spheres is more difficult in contrast to the cantilever because the appearance of the spheres change during the handling (Fig. 3). The cantilever shadows the spheres due to position changes, which modifies the intensity and contrast of the image. Due to the view angle depended detector shadowing effects and different brightness and texture features of the spheres, it was decided to work on edge images to generate reliable results. 


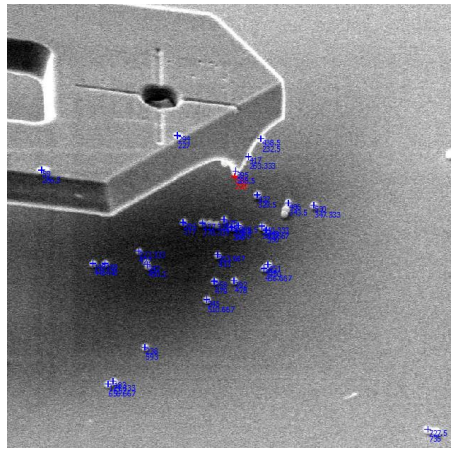

(a) Cantilever with the FIB structured tracking mark and detected melanin spheres.

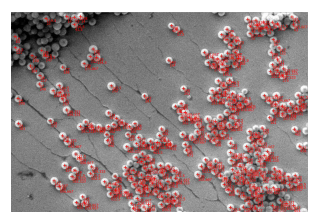

(b) 273 spheres were detected without any fail detection.

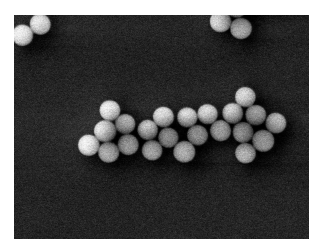

(c) SEM image of melanin spheres with different magnifications and view angles.
Fig. 3. These images show the diversity of image characteristics of the spheres and the related detection problems (shadowing, contrast and intensity modifications, ....).

The CC is modified to use specially generated templates. After applying the edge detection filter a $\mathrm{CC}$ is used to find the spheres. In contrast to normal $\mathrm{CC}$ binary ring templates are used. These ring templates are specially generated to fit to the contour of a circle, independently of the texture values of the sphere. Their radii depend on the diameter of the spheres, which minimum and maximum values are supposed to be known from the product specifications. Therefore, a list of ring templates with different radii and ring widths are automatically generated and passed to the $\mathrm{CC}$. The number of $\mathrm{CC}$ cycles is equal to the number of ring templates. The result list contains all spheres of the cycles with a correlation result higher than the defined threshold for the $\mathrm{CC}$. At the end, the result list is clustered and the weighted center points are calculated. The clustering is necessary because the same sphere can be detected multiple on a slightly different position in each $\mathrm{CC}$ cycle.

To meet the teleoperation requirements, the detection should be fast. However, in order to avoid a slowdown of the cantilever tracking, the positions of the melanin spheres are calculated with a low priority in the Olvis framework. This choice was made since the cantilever is moved more often than the spheres. The sphere tracking has an update rate of 3 to $8 \mathrm{~Hz}$ for searching in the sphere diameters in the range of 1 to $1.6 \mu \mathrm{m}$ with an image size of $627 \times 627$ pixels. This tracking algorithm, as well as the one for the cantilever detection, have a sub-pixel accuracy.

During this application the involved objects were only tracked in $\mathrm{x}$ and $\mathrm{y}$ directions. The relative height of the cantilever with respect to the substrate is thus unknown. For this work, this lack of information is compensated by the force measurement, which estimates the vertical force applied on the cantilever and enables to determine if the tip is or not in contact with the substrate or an object.
Other solutions might include a calibration step, or image processing based on the distance between the tip and its shadow on the substrate.

\section{INTUITIVE TELEOPERATION ENVIRONMENT}

To provide an intuitive environment of manipulation, both visual and haptic feedback are proposed. They are based on both force measurement and vision tracking of the tool and the objects.

\section{A. Virtual Reality Scene}

To fit haptic feedback requirements, communication delays and thus the amount of data to transfer should be limited to strict minimum. Vision data represents the heaviest load on the network. In the case studied here where the distance between the operator and the manipulator is several hundreds kilometers, the synchronicity between the seeing and handling can only be guaranteed by avoiding the communication delay. This is ensured by an efficient software architecture (see Sec. III) and a drastic reduction on the size of data packets. The approach proposed here avoids to send over the complete camera output and replaces it with an immersive virtual reality scene, presented in stereoscopic 3D. Instead of transmitting the complete image, only the cantilever's and the closest object's positions are sent over using respectively the sensor feedback of piezo actuators and vision tracking. Considering that this data represents only $2 \times 3 \times 64$ bits (doubles), the gain compared to the transmission of a full frame video image is enormous. In addition to save transmission bandwidth, this approach also replaces the SEM image which lacks perspective and intuitive depth information by an easier-to-interpret 3D reproduction.

The virtual reality scene of the micromanipulator is constructed using Blender, an open source 3D content creation suite [22]. It is easily extensible using Python programming language, includes Bullet real-time physics engine and can produce stereoscopic output. The scene includes the AFM tip used as the manipulation tool and a sphere as the manipulated object. In that work, it was decided to represent only the sphere closest to the cantilever tip. This facilitates the manipulation for untrained users by displaying only important information on the reconstructed scene. The scene is constructed setting 1 Blender unit as $1 \mu \mathrm{m}$ and is depicted in Fig 1. All along the operation, the user has complete freedom to modify the camera position and zoom.

The drawback of this method is the requirement of prior knowledge of geometries and dimensions and an initial calibration process between real image and virtual reality frames. As in this case the cantilever's and manipulated objects' geometries are perfectly known and their virtual models are created prior to the operation, it is sufficient to map their real positions to the virtual scene. Details such as the precise shape of the objects, or their exact dimensions are not represented in the virtual scene. However, this information is not necessary for a manipulation task. 
In order to calibrate the real and the virtual cantilever positions, the real cantilever is moved to the contact with substrate, then its vertical position is adjusted such as its flexion is null, without snapping the tip off the surface. This position is offset as the $(0,0,0)$ on the virtual scene. The object position is obtained from image processing along with the tip position. As all the projection lines in a SEM image are parallel, the resulting image is an orthographic projection. Considering that all the objects are on the same plane, i.e lying on the substrate where the angle of substrate plane and image plane is known, their respective 3D positions can be calculated with fixed $z$. The tip and object image positions is then used to calibrate those initial positions with the reference positions of the virtual scene.

During the manipulation and after the calibration, the VR engine receives periodically positions of the cantilever and the manipulated object and updates them respectively. If a delay in communication appears such as the VR frame rate is higher than the data transmission rate, the included Bullet physics engine extrapolates the positions. This extrapolation is not expected to reproduce faithfully the real physics. As a new updated position is received the object jumps to the position, the Bullet engine minimizes this behavior and leads to a smoother user experience. Also note that over several experiences, this extrapolation approach was called upon extremely rarely.

\section{B. Haptic Feedback}

In addition to 3D display of the scene, haptic feedback is proposed to provide intuitive manipulation tools. Users control the cantilever through a haptic interface, and feel forces which give them information about the scene. The haptic coupling connects the haptic device to the manipulation tool (Fig. 4). Users interact with the master device by applying a force $\mathbf{F}_{\mathbf{o p}}$ on it to control the slave device. The desired position of the tip $\mathbf{P}_{\mathbf{t}}$ is set using the position of the haptic handle $\mathbf{P}_{\mathbf{h}}$, scaled by a factor $\alpha_{p}$. Since the workspace of this arm is limited, a clutching function has been implemented so that users can set the haptic handle to a desired position without moving the cantilever [23]. Operators feel a haptic force $\mathbf{F}_{\mathbf{h}}$ derived from both force measurements and the vision algorithm.

Force measurements, obtained by the flexion of the AFM probe, are used to compute the vertical haptic force $f_{h}^{z}$ transmitted to users. It is computed from the vertical force $f_{t}$ applied on the tip, scaled by a factor $\alpha_{f}: f_{h}^{z}=\alpha_{f} f_{t}$.

To compute the haptic force in the $(x, y)$ plane (substrate plane), vision algorithms are used. They enable to determine the respective position of the tool $\left(\mathbf{P}_{\mathbf{t}}\right)$ and the sphere $\left(\mathbf{P}_{\mathbf{s}}\right)$. This force is based only on the distance to the closest sphere. Indeed, simplifying the scene enables the user to concentrate on the manipulation task. Other scenario considering all the objects in a close neighborhood of the tool could be proposed.

As explained in Section IV, the vectors $\left.\mathbf{P}_{\mathbf{t}}\right)$ and $\mathbf{P}_{\mathbf{s}}$ are of dimension $2 \times 1$ in the $(x, y)$ plane. Based on that information, a repulsive haptic force is computed. This repulsion would

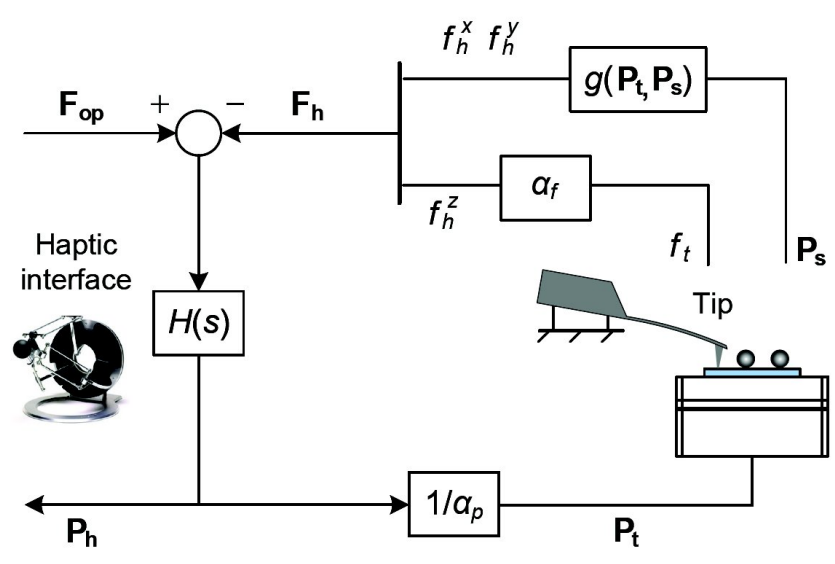

Fig. 4. Haptic coupling.

push away the tool from the spheres in the horizontal plane and avoid involuntary contact. This force is based on the distance between the tool and the object:

$$
\mathbf{D}=\left[\begin{array}{l}
d^{x} \\
d^{y}
\end{array}\right]=\left[\begin{array}{l}
p_{t}^{x}-p_{s}^{x} \\
p_{t}^{y}-p_{s}^{y}
\end{array}\right]
$$

where $\|\mathbf{D}\|=\sqrt{d^{x^{2}}+d^{y^{2}}}$ is the distance between the sphere and the tip, and $d^{x}$ (resp. $d^{y}$ ) are the coordinates along the $x$ (resp. y) axis. Based on that distance, a repulsive force field is computed. While the distance $\|\mathbf{D}\|$ is greater than a given threshold $R_{\text {int }}$, the force is null since it is considered that the tip is far enough from the sphere. When this distance becomes less than the threshold, a non null repulsive force $f_{r}$ is computed:

$$
f_{r}= \begin{cases}\left(1.0-\frac{\|\mathbf{D}\|}{R_{\text {int }}}\right) f_{\max } & \text { if }\|\mathbf{D}\|<R_{\text {int }} \\ 0 & \text { else }\end{cases}
$$

where $f_{\max }$ is the maximum force that would be sent for $\|\mathbf{D}\|=0$. This value will never be reached since the minimum distance between the tip and the center of the sphere cannot be less then the radius of the object. This force enables users to know when they are entering the interaction radius, and to estimate the relative position of the tip and the sphere for spheres of a given dimension. Moreover, by setting the threshold value equal or slightly superior to the radius of the object, it would also be possible to let the user feel solely the contact in the horizontal plane between the tip and the object.

The force sent to the haptic device is computed by projecting $f_{r}$ along the $x$ and $y$ axes, proportionally to $d^{x}$ and $d^{y} . f_{h}^{z}$ is given by measurements of the tip deflection as explained above:

$$
\mathbf{F}_{\mathbf{h}}=\left[\begin{array}{c}
f_{h}^{x} \\
f_{h}^{y} \\
f_{h}^{z}
\end{array}\right]=\left[\begin{array}{c}
\frac{d^{x}}{\|\mathbf{D}\|} f_{r} \\
d^{y} \\
\|\mathbf{D}\| \\
\alpha_{f} f_{t}
\end{array}\right]
$$

If the tip is not in contact with the substrate $\left(f_{t}=0\right)$ the haptic feedback is null. In that work, it is considered that if the tip is above the substrate, users cannot collide 
with spheres. Alternatively, a given height under which force feedback is activated could be defined to take into account the sphere height. This height could be determined in realtime using vision information, such as the distance between the tip and its shadow.

\section{EXPERIMENTAL RESULTS}

To validate the tools developed, experiments are conducted. The first task performed is in an approach-retract experiment. It consists in approaching the cantilever towards the substrate, making contact, applying a force on the substrate, and then retracting. This is used for educational purposes, to give users a better understanding of nanoscale phenomena [24], or to perform indentation or patterning tasks [25]. In this work, this simple 1D task enables to test the robotic control architecture and the stability of the haptic feedback. There is no display of the virtual scene. Vision algorithms are not used since only the vertical force is of interest: the transpose of the haptic force is computed as $\mathbf{F}_{\mathbf{h}}^{T}=\left[\begin{array}{lll}0 & 0 & \alpha_{f} f_{t}\end{array}\right]$.

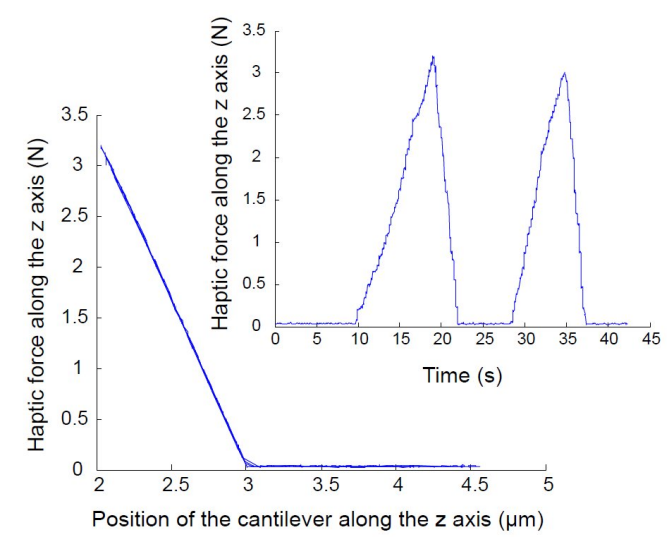

Fig. 5. Approach-retract experiment. Scaling factors are set to: $\alpha_{p}=4800$, $\alpha_{f}=1.10^{6}$.

Results are depicted in Fig. 5. The haptic force is represented as a function of time and as a function of the position of the cantilever. The system remains stable for that experiment. The haptic feedback enables users to know when the cantilever is in contact with the substrate, and to estimate the effort applied on the substrate since the haptic force is proportional to the flexion (hence the vertical displacement) of the cantilever.

Before performing manipulations of objects, it is necessary to get information about their position with respect to the tool. A visual display of the 3D reconstructed virtual reality scene is provided (Fig. 6). Repulsive haptic force field are also transmitted to users based on measurements from the tip deflection combined with vision algorithms (Eq . 3).

Results are depicted in Fig. 7. The position of the sphere as well as the trajectory of the tip are represented. Haptic force on the plane of the substrate is given for several points. This force tends to keep the tip away from the spheres, and enable users to localize precisely the objects. For the sake of clarity,

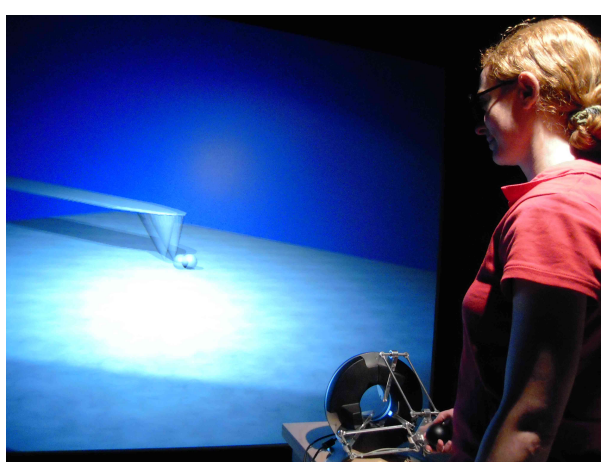

Fig. 6. Teleoperation of microspheres using a haptic interface, with 3D virtual reconstruction of the manipulation scene. This image is presented in stereoscopic 3D to the user who can move the camera at will.

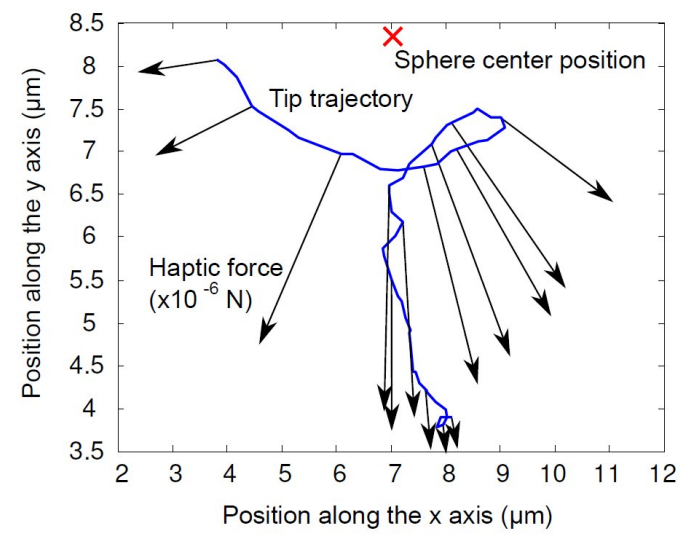

Fig. 7. Sphere avoidance: repulsive haptic force field. Haptic coupling gains for the force computation are set to: $R_{\text {int }}=5 \mu \mathrm{m}, f_{\max }=4 \mathrm{~N}, \alpha_{f}=1.10^{6}$ and $\alpha_{p}=4800$. The magnitude of the force vectors is proportional to the distance between the tip and the object (note that $x$ and $y$ scales are different).

the vertical force is not depicted. It is similar to the one of the approach-retract experiment. Performances of the system are summarized in Table I.

TABLE I

PERFORMANCES OF THE SYSTEM

\begin{tabular}{ll}
\hline Item & Performances \\
\hline Transmission & RTT: $36-39 \mathrm{~ms}$, average: $37 \mathrm{~ms}$ \\
Vision tracking & $\begin{array}{l}\text { sphere: } 3-8 \mathrm{~Hz} \\
\text { cantilever: } 50 \mathrm{~Hz}\end{array}$ \\
Virtual reality & $\begin{array}{l}\text { 3D visual reconstruction } \\
\text { data load: } 2 \times 3 \times 64 \text { bits } \\
\text { force measurement: contact tool/substrate } \\
\text { Haptic }\end{array}$ \\
& vision: distance tool/object
\end{tabular}

The developed tools enable the intuitive manipulation of an AFM integrated into an SEM from a remote site, that can be situated hundreds of kilometers from the setup. Both visual and haptic feedback are provided.

\section{CONCLUSION}

In that work, new tools have been developed to enable intuitive manipulation of microscale objects from geographically distant sites. Ease of manipulation is ensured by visual 
and haptic feedbacks. Force measurements are limited while using a cantilever simultaneously as the manipulation and sensing tool. Therefore vision detection and robust tracking algorithms with respect to SEM image issues, such as shadowing, are used to get additional information about the scene. They enable the 3D reconstruction of the manipulation area, displayed in stereoscopic view. Haptic feedback is based on both the vision tracking and force measurements. Stability of haptic feedback is addressed by minimizing delays using efficient vision algorithms and robotic control architecture. The modularity of the control system also enables a wide variety of applications.

To widen the range of applications, nanometer size objects should be considered. Other equipments, such as grippers, should also be integrated, and associated strategies to derive intuitive haptic feedback and calibration of the virtual scene should be proposed. Next step will consist in moving the spheres, for example by pushing them with the cantilever. The same visual and haptic feedback will be used, but a special care will be taken to define the manipulation strategy. Several issues, such as sticking effects, must be addressed to perform this manipulation [26].

This work is a first step towards teleoperation systems enabling intuitive manipulation of micro or nanoscale objects between geographically distant sites. These systems will offer great opportunities for researchers, giving them access to rare equipments.

\section{ACKNOWLEDGMENTS}

Parts of this work have been funded by the European project FIBLYS and by the project PROCOPE (DAADEgide). Special thanks to Uwe Mick for the technical and scientific support.

\section{REFERENCES}

[1] A. Muhammad, S. Esque, J. Mattila, M. Tolonen, P. Nieminen, O. Linna, M. Vilenius, M. Siuko, J. Palmer, and M. Irving, "Development of water hydraulic remote handling system for divertor maintenance of ITER," in IEEE Symposium on Fusion Engineering, 2007, pp. 1-4.

[2] L. Peñín, K. Matsumoto, and S. Wakabayashi, "Force reflection for time-delayed teleoperation of space robots," in IEEE International Conference on Robotics and Automation, vol. 4, 2000, pp. 3120-3125.

[3] J. Marescaux, J. Leroy, F. Rubino, M. Smith, M. Vix, M. Simone, and D. Mutter, "Transcontinental robot assisted remote telesurgery: feasibility and potential applications," Annals Of Surgery, vol. 235, no. 4, pp. 487-492, 2002.

[4] M. Guthold, M. Falvo, W. Matthews, S. Paulson, S. Washburn, D. Erie, R. Superfine, F. Brooks Jr., and R. Taylor II, "Controlled manipulation of molecular samples with the nanoManipulator," IEEE/ASME Transactions on Mechatronics, pp. 189-198, 2000.

[5] I. Bukusoglu, C. Basdogan, A. Kiraz, and A. Kurt, "Haptic manipulation of microspheres using optical tweezers under the guidance of artificial force fields," Presence: Teleoperators and Virtual Environments, vol. 17, no. 4, pp. 344-364, 2008.

[6] M. Ammi and A. Ferreira, "Robotic assisted micromanipulation system using virtual fixtures and metaphors," in IEEE International Conference on Robotics and Automation, 2007, pp. 454-460.

[7] S.-G. Kim and M. Sitti, "Task-based and stable telenanomanipulation in a nanoscale virtual environment," IEEE Transactions on Automation Science and Engineering, vol. 3, no. 3, pp. 240 - 247, 2006.
[8] M. Sitti and H. Hashimoto, "Teleoperated touch feedback from the surfaces at the nanoscale: modeling and experiments," IEEE/ASME Transactions on Mechatronics, vol. 8, no. 2, pp. 287-298, 2003.

[9] G. Li, N. Xi, M. Yu, and W.-K. Fung, "Development of augmented reality system for AFM-based nanomanipulation," IEEE/ASME Transactions on Mechatronics, vol. 9, no. 2, pp. 358-365, 2004.

[10] W. Vogl, B. Ma, and M. Sitti, "Augmented reality user interface for an atomic force microscope-based nanorobotic system," IEEE Transactions on Nanotechnology, vol. 5, no. 4, pp. 397-406, 2006.

[11] C. D. Onal and M. Sitti, "Teleoperated 3-D force feedback from the nanoscale with an atomic force microscope," IEEE Transactions on Nanotechnology, vol. 9, no. 1, pp. 46-54, 2010.

[12] A. Bolopion, B. Cagneau, and S. Régnier, "2D micro teleoperation with force feedback," in Proceedings of the IEEE International Conference on Intelligent Robots and Systems, 2009, pp. 3265-3570.

[13] A. Bolopion, H. Xie, S. Haliyo, and S. Régnier, "Haptic teleoperation for 3D microassembly of spherical objects," to be published in IEEE/ASME Transaction on Mechatronics, 2010.

[14] T. Sievers and S. Fatikow, "Real-time object tracking for the robotbased nanohandling in a scanning electron microscope," Journal of Micromechatronics - Special Issue on Micro/Nanohandling, vol. 3, no. 3-4, pp. 267-284(18), 2006.

[15] M. Boukhnifer and A. Ferreira, "Wave-based passive control for transparent micro-teleoperation system," Robotics and Autonomous Systems, vol. 54, no. 7, pp. 601-615, 2006.

[16] U. Mick, V. Eichhorn, T. Wortmann, C. Diederichs, and S. Fatikow, "Combined nanorobotic AFM/SEM system as novel toolbox for automated hybrid analysis and manipulation of nanoscale objects," in IEEE International Conference on Robotics and Automation, 2010, pp. 4088-4093.

[17] C. Stolle, "Distributed control architecture for automated nanohandling," in International Conference on Informatics in Control, Automation and Robotics (ICINCO'07), 2007, pp. 127-132.

[18] A. Bolopion, B. Cagneau, S. Haliyo, and S. Régnier, "Analysis of stability and transparency for nanoscale force feedback in bilateral coupling," Journal of Micro - Nano Mechatronics, no. 4, pp. 145$158,2009$.

[19] J. Jasper, C. Edeler, C. Diederichs, M. Naroska, C. Stolle, and S. Fatikow, "Towards automated robotic nanomanipulation systems," IEEE/ASME International Conference on Advanced Intelligent Mechatronics, 2009.

[20] T. Wortmann, C. Dahmen, R. Tunnel, and S. Fatikow, "Image processing architecture for real-time micro- and nanohandling applications," Proc. of the Eleventh IAPR Conference on Machine Vision Applications (MVA), 2009.

[21] A. Goshtasby, S. Gage, and J. Bartholic, "A two-stage cross-correlation approach to template matching," IEEE Trans. Pattern Analysis and Machine Intelligence, vol. 6, no. 3, pp. 374-378, 1984.

[22] Blender, http://www.blender.org/.

[23] L. Dominjon, A. Lécuyer, J.-M. Burkhardt, G. Andrade-Barroso, and S. Richir, "The "Bubble" technique: interacting with large virtual environments using haptic devices with limited workspace," in Proceedings of the First Joint Eurohaptics Conference and Symposium on Haptic Interfaces for Virtual Environment and Teleoperator Systems, 2005, pp. 639-640.

[24] S. Marliere, D. Urma, J. Florens, and F. Marchi, "Multi-sensorial interaction with a nano-scale phenomenon: The force curve," in Proceedings of the Eurohaptics, 2004, pp. 246-252.

[25] C. D. Onal and M. Sitti, "A scaled bilateral control system for experimental one-dimensional teleoperated nanomanipulation," The International Journal of Robotics Research, vol. 28, no. 4, pp. 484497, 2009.

[26] C. Onal and M. Sitti, "Visual servoing-based autonomous 2-D manipulation of microparticles using a nanoprobe," IEEE Transactions on Control Systems Technology, vol. 15, no. 5, pp. 842-852, 2007. 